\title{
LA PARTICIPACIÓN: UNA RESPUESTA AL PROBLEMA ÉTICO DE LA RELACIÓN ENTRE TECNOLOGÍA Y SOCIEDAD \\ Gabriela Bernal*
}

Hasta cierto punto nuestra inteligencia es la medida de nuestra responsabilidad, y desde el momento en que la ciencia y la tecnología fueron percibidas colectivamente, se convirtieron en la medida de la responsabilidad pública.

Javier Ordóñez ${ }^{1}$

\section{Introducción}

$\mathrm{E}_{1 \text { término desarrollo ha evolucio- }}$ nado a través del tiempo, pasando desde una concepción limitada a factores económicos surgidos a partir de los intereses y condiciones de las sociedades desarrolladas de Occidente, hasta una concepción que incluye variables con un trasfondo ético y humano. Un variable común en estas concepciones es el desarrollo tecnológico, aspecto que en la mayoría de los casos, el enfoque de análisis se ha limitado a describir en términos de progreso tecnológico y su impacto en la economía de un país, restando importancia a los beneficios sociales que dicho desarrollo tecnológico ha aportado.

El objetivo de este ensayo es analizar el problema de la relación entre sociedad y tecnología y bosquejar algunas propuestas. A lo

* Tecnológico de Monterrey, campus Toluca.

${ }^{1}$ Javier Ordóñez, Ciencia, Tecnología e Historia, 2003, México, FCE, p. 86. 
largo del análisis se definirá como argumento central que la búsqueda del bienestar de las sociedades es lo que debe guiar el desarrollo tecnológico. Las dos preguntas que se atienden son las siguientes: (i) ¿Cómo puede definirse el desarrollo tecnológico para que coincida con los intereses de las sociedades humanas? (ii) ¿Existe realmente capacidad de decisión en las sociedades para orientar el desarrollo tecnológico en una dirección acorde con los intereses de las sociedades humanas?

Estas interrogantes nos llevan a la necesidad de dar un enfoque ético al estudio del tema de la tecnología, considerando la importancia de dar a la sociedad las facultades para participar en las decisiones acerca de qué tecnología puede ser la más adecuada para ella. El análisis aportará los elementos que nos permitirán sentar las bases para estructurar un modelo de desarrollo en el cual la tecnología y la participación social sean el eje central de dicho modelo.

\section{El concepto de desarrollo}

32 El concepto de desarrollo que ha predominado desde la escuela clásica liberal se encuentra ligado a nociones como riqueza, evolución y progreso. En la actualidad éste suele ser entendido ya sea como crecimiento, como etapa o como proceso de cambio estructural global. El desarrollo como crecimiento considera para su medición indicadores tales como el PIB, PNB o el ingreso per cápita. En un trabajo presentado a solicitud de la UNESCO, François Perroux (1984) opinaba que esta noción de crecimiento presentaba varias deficiencias; primero, existen problemas de medición que se incrementan en los países subdesarrollados que presentan amplios sectores atrasados desvinculados del sector moderno de la economía. En segundo lugar, el concepto de crecimiento ocultaba los efectos de la destrucción ecológica y el deterioro de los productores directos; también evitaba discutir las condiciones reales de vida de la mayoría de la población o la distribución de ingreso entre las distintas clases y grupos sociales. 
En la etapa del modelo primario exportador que vivió América Latina más o menos hasta la depresión de la década de los treinta era claro que el crecimiento beneficiaba al sector exportador moderno, generalmente controlado por capital extranjero, y que la capacidad de transmisión de dicho crecimiento al resto del sistema productivo era mínima. A partir de la década de los setenta, con el modelo neoliberal, esa historia se repite con el agravante de que el crecimiento es nulo.

En la década de los cincuenta, predomina la teoría universal de las cinco etapas, postulada por W. Rostow, quien precisa la corriente neo-evolucionista liberal de Estados Unidos, donde uno de los factores principales para entender el desarrollo de un país es el crecimiento económico.

Sin embargo, en ninguna de estas etapas se respondían preguntas como: ¿crecimiento para qué? ¿en qué condiciones el crecimiento es provechoso? ¿el crecimiento es provechoso para algunos miembros de la sociedad o para todos?

Ante esta tendencia liberal surge la corriente estructuralista del desarrollo económico representada por la Comisión Económica para la América Latina (CEPAL), que entiende el desarrollo como resultado de las relaciones estructurales entre los factores sociales, culturales, políticos y económicos de una sociedad determinada y su interacción con otras sociedades. Esta corriente exponía, a partir de la década de los cincuenta, el modelo centro-periferia en el cual se explicaba la desigualdad estructural en las relaciones de los países capitalistas industrializados y los denominados dependientes, así como la incuestionable hegemonía del centro.

De acuerdo con Paul Baran ${ }^{2}$

La irrupción del capitalismo occidental en los hoy países subdesarrollados al precipitar con irresistible energía algunas de las condiciones básicas para el desarrollo de un sistema capitalista, bloqueó con igual

${ }^{2}$ Citado en la II Conferencia Internacional Red de Estudios sobre el Desarrollo Celso Furtado, mayo 2004. 
fuerza el crecimiento de las otras. [...] Aunque la expansión de la circulación de mercancías, la pauperización de un gran número de campesinos y artesanos y el contacto con la técnica occidental dio un impulso al desarrollo del capitalismo, este desarrollo fue deformado y mutilado para que se adaptase a los objetivos del imperialismo occidental.

El desarrollo no es únicamente un proceso de acumulación de capital y de progreso técnico como lo concibe la teoría neoclásica de crecimiento, sino un proceso de cambio social y de reorganización institucional. Para Hoff y Stiglitz, en los enfoques neoclásicos:

La repartición de las riquezas no cuenta más, desde el momento en que no se interesan más que en la eficacia. Esa es su hipótesis fuerte. Dejando de lado las instituciones, la historia y las cuestiones de la repartición, la economía neoclásica deja de lado lo que constituye el corazón mismo de la economía del desarrollo. ${ }^{3}$

Y así como la teoría neoclásica deja de lado las instituciones, también se olvida de la dimensión cultural, social y antropológica del desarrollo, así como sus nexos indisolubles con el desarrollo de 34 la democracia, entendida ésta no sólo como la acción de votar sino como proceso de participación y organización popular.

El problema del desarrollo visto desde esta última perspectiva es el que proyectó la cuestión ética del desarrollo como una preocupación fundamental para su análisis. Las teorías éticas del desarrollo pretenden dar una respuesta al asunto de qué debe entenderse por buen desarrollo o qué debe entenderse como desarrollo auténticamente humano. El desarrollo, en este sentido, no constituye un fin en sí mismo sino que es un prerrequisito del progreso social.

Como afirma Amartya $\mathrm{Sen}^{4}$ el desarrollo puede concebirse como un proceso de expansión de las libertades reales de que disfrutan los

${ }^{3}$ Joseph Stiglitz, "El Rumbo de las reformas. Hacia una Nueva Agenda para América Latina”, en Revista de la CEPAL, ${ }^{\circ}$ 80, agosto, 2003, p. 322.

${ }^{4}$ Amartya Sen, Desarrollo y libertad, 2000, Barcelona, Planeta. 
individuos. El hecho de centrar la atención en las libertades humanas contrasta con las visiones más estrictas del desarrollo, como su identificación con el crecimiento del PNB, con el aumento del PIB, con la industrialización, con los avances tecnológicos o con la modernización social.

La libertad también depende de otros determinantes, como las instituciones sociales y económicas (por ejemplo, los servicios de educación y de atención médica), así como los derechos políticos y humanos (entre ellos, la libertad para participar en debates y escrutinios públicos) $[\ldots]$ La concepción del desarrollo como un proceso de expansión de las libertades fundamentales lleva a centrar la atención en los fines por los que cobra importancia el desarrollo y no sólo en algunos de los medios que desempeñan entre otras cosas, un destacado papel en el proceso. ${ }^{5}$

La obtención de esos mínimos, que actualmente son conocidos como derechos sociales del hombre dentro de la Declaración Universal de los Derechos Humanos, no es el resultado del desarrollo económico, que si se deja libre en su propia dinámica tiende a generar desigualdad y concentración de la riqueza, sino que requiere además la acción del Estado y la organización de la sociedad civil. Si bien el progreso social reclama un crecimiento del producto nacional y de cambios cualitativos en la estructura productiva, también requiere de la existencia de instituciones y de la acción organizada de los grupos sociales. El supuesto del 'goteo', es decir la noción de que el crecimiento económico redundará, tarde o temprano, en progreso social, se está revelando como falsa en nuestros días, como lo evidencian diversas experiencias históricas.

${ }^{5}$ Ibid., p. 19. 


\section{El desarrollo tecnológico}

La preocupación en la relación entre crecimiento económico y desarrollo tecnológico toma fuerza a partir de la Segunda guerra mundial. ${ }^{6}$ Sin embargo, como comentamos en el apartado anterior, los análisis se realizaban en virtud de los efectos que provocaba en las variables consideradas importantes: crecimiento, comercio, empleo, producción etc. Pero el análisis de las condiciones que provocan el avance tecnológico y la difusión de innovaciones para luego discutir sus efectos no se desarrolla hasta fines de la década de los cincuenta.

A pesar de la preocupación originaria de la CEPAL por la distribución de los frutos del progreso técnico, el esfuerzo analítico no se centró en determinar la lógica del progreso técnico, sino que se equiparó con industrialización y luego se estudiaron sus consecuencias distributivas en el comercio internacional y en la conformación de estructuras nacionales. En esa corriente, las preocupaciones metodológicas giraron en torno de la crítica de la dependencia ante las consecuencias del desarrollo periférico, y en este sentido la tecnología se consideraba sólo uno de los aspectos que contribuía a la descripción. Luego, con el retorno de la agenda neoclásica, que desplazó a la corriente de la CEPAL, el papel del desarrollo tecnológico tampoco figuró entre las preocupaciones analíticas del desarrollo.

Si a pesar de la preocupación que suscita la tecnología apenas encontramos filósofos, politólogos o sociólogos que conviertan a la tecnología en objeto de pensamiento, quizás una aventurada respuesta es que la velocidad y profundidad del proceso de transformación que la tecnología causa en las formas culturales haya opacado el 'sentido de los cambios'. Broncano ${ }^{7}$ dice que a estas dificultades habría que

${ }^{6}$ Ennio Rodríguez, "La endogenización del cambio tecnológico: un desafío para el desarrollo", en El Desarrollo desde dentro. Un enfoque neoestructuralista para América Latina, 1995, México, FCE, p. 282.

${ }^{7}$ Fernando Broncano (ed.), Nuevas meditaciones sobre la técnica, 1995, Madrid, Trotta. 
añadir la actitud distante y extrema de muchos intelectuales, que ven en la técnica la madre de todos los males o, por el contrario, de todas las soluciones a los males que nos aquejan, y lo precisa de la siguiente manera:

Pertenece a esta actitud externalista la consideración de la tecnología como una caja negra de la que sólo interesan los productos y/o las consecuencias de los productos, pero no los métodos de trabajo, la especial forma de su conocimiento, el modo en el que se articulan los factores sociológicos y económicos con los intereses estrictos de la investigación. ${ }^{8}$

Esta razón explica el interés de este ensayo por una perspectiva más amplia en relación a la metodología y el análisis del desarrollo tecnológico en las últimas décadas.

Para Quintanilla, ${ }^{9}$ los factores que influyen en el desarrollo tecnológico son de un carácter que podemos llamar 'interno' (cuando atienden aspectos como mejora de la eficiencia de un proceso, de la duración de una máquina o de la fiabilidad de un dispositivo), o 'externo' (que incluye factores sociológicos, demográficos, económicos, culturales, etc.). Los criterios denominados externos se refieren al valor de la tecnología para la sociedad que se propone usarla o desarrollarla.

La evaluación externa de tecnologías es esencial ya que un proyecto tecnológico puede ser factible, sumamente eficiente, efectivo y fiable y, sin embargo, ser inexplicable e irrelevante para una comunidad. Las restricciones pueden ser el costo, si es útil o inútil para los propósitos del grupo, perturbador de la estructura social o del medio ambiente, arriesgado o contrario a sus principios; si fuera el caso de que la evaluación fuera sólo interna, el proyecto quedaría relegado a las 'buenas intenciones' que nunca llegaron a ser realidad. Al respecto, Quintanilla dice que "las demandas, las necesidades o los deseos de una sociedad condicionan los objetivos de desarrollo tecnológico

${ }^{8}$ Ibid., p. 11.

${ }^{9}$ Ibid., p. 13. 
tanto como las disponibilidades de recursos materiales, científicos y tecnológicos previos". ${ }^{10}$

De acuerdo con lo anterior, si bien es importante definir el concepto de desarrollo tecnológico con base a criterios internos de eficiencia, también es importante tomar en cuenta la forma concreta en que se desenvuelve la tecnología dentro de los grupos sociales y el valor que éstos asignen a los diferentes objetivos posibles. La evaluación externa de tecnologías se debe situar en el centro de las reflexiones sociales y plantea problemas de carácter metodológico, organizacional y político.

La importancia actual de la evaluación externa de tecnologías está justificada. En primer lugar, porque las tecnologías de hoy afectan a toda la sociedad de múltiples formas y sobre todo a las posibilidades futuras de desarrollo económico, social y cultural de la humanidad. En segundo lugar, porque el cambio tecnológico es muy rápido y se hace cada vez más necesario prever las consecuencias que la implantación de una tecnología puede tener para el futuro.

En tercer lugar, porque hemos llegado a convencernos de que el desarrollo tecnológico depende de decisiones humanas y de que tal desarrollo se puede orientar en múltiples direcciones, de acuerdo con nuestros intereses, o en contra de ellos. ${ }^{11}$

Broncano, ${ }^{12}$ al igual que Quintanilla retoma la idea de que los valores bajo los que cabe discutir un proyecto tecnológico son internos y externos y añade al análisis la percepción que los diferentes actores sociales involucrados en el desarrollo tecnológico, pueden tener de estos valores. Sostiene que el predominio de valores externos puede ser visto por los ingenieros y científicos como una 'interferencia' en el desarrollo normal del proceso autónomo de la tecnología y que el predominio de valores externos, consecuencia del hecho de que la tecnología sobre-

${ }^{10}$ Miguel Ángel Quintanilla, Tecnología: un enfoque filosófico y otros ensayos de filosofía de la tecnología, 2005, México, FCE, p. 139.

${ }^{11}$ Ibidem, p. 140.

${ }^{12}$ Fernando Broncano (ed.), op. cit. 
vive a causa de su utilidad económica y social, tiende a ser visto por los agentes sociales implicados, como resultado de un 'dominio de tecnócratas' quienes estarían generalizando injustificadamente criterios de eficiencia internos a campos en los que ya no son actores legítimos en la decisión. Si esta situación la analizamos en el marco de una empresa, es lógico que las propuestas de los departamentos técnicos de Investigación y Desarrollo se subordinen a otros departamentos. Sin embargo, en el marco de la sociedad los criterios no son tan claros: las decisiones en esta materia son complicadas debido a la gran cantidad de variables involucradas, como intereses de grupo, necesidades, cultura, modificación del medio ambiente, políticas públicas, y a la compleja interrelación entre ellas. Para Broncano la evaluación de la tecnología es uno de los campos en los que la perspectiva filosófica puede servir de ayuda, sea en el análisis y dilucidación de conceptos, sea en la propuesta positiva de criterios.

Es urgente comenzar estudios que nos ayuden a conocer los valores implicados en las decisiones tecnológicas ya desde los primeros momentos de formación de las tecnologías. ${ }^{13}$

De las propuestas hasta aquí descritas para motivos de este análisis nos limitaremos a destacar la importancia de la evaluación externa y dentro de ésta las consecuencias sociales de la aplicación de una tecnología. En el caso de una tecnología disponible, de lo que se trata es de valorar las consecuencias que pueda tener su aplicación por parte del grupo social en circunstancias concretas. El problema principal que aquí se presenta es la amplitud de variables que hay que considerar al reflexionar sobre las tecnologías y a la ausencia de un punto de referencia estable (cualquier tecnología de cierta importancia terminará alterando en mayor o menor medida la estructura social, las costumbres, la vida cotidiana, etc.). Ante las pocas posibilidades de prever las consecuencias de la tecnología, consideramos que es preciso establecer

${ }^{13}$ Ibid., p. 13. 
mecanismos de participación que incluyan el diálogo con los usuarios y posibles afectados en la política de desarrollo tecnológico. No parece razonable seguir limitando la reflexión a la aplicación de técnicas de cálculo; la propuesta es centrar la atención en los procesos de participación del conjunto de la sociedad en la evaluación tecnológica y en la adopción de decisiones. En este sentido, la dimensión política ${ }^{14}$ de esta forma de evaluación externa es fundamental. ${ }^{15}$

\section{Relación entre desarrollo, tecnología y sociedad y/o democratización del desarrollo tecnológico}

Una primera propuesta para la solución a este problema es dar a la sociedad el conocimiento para tener un papel protagónico al decidir qué tecnología puede ser mejor y más útil para el progreso de su comunidad. La propuesta es diseñar mecanismos para involucrar a la sociedad en los desarrollos tecnológicos para lograr que encuentren sentido a la aplicación de la tecnología en su quehacer social. Esta idea la apoyamos en el siguiente argumento de Broncano:

La sociedad establece sus necesidades en la medida del conocimiento que tiene de sus posibilidades, y este conocimiento se lo proporciona en una gran medida las expectativas de las comunidades científicas. ${ }^{16}$

${ }^{14}$ Cuando nos referimos a dimensión política no nos restringimos únicamente al proceso que conllevan las políticas públicas y las decisiones que emanan de los proyectos nacionales de desarrollo, sino a una concepción más amplia en la cual se incluye la participación social como una parte sustancial de la democratización en las decisiones que tienen que ver con el uso de la tecnología.

${ }^{15}$ El Informe de la OCDE (1979, citado en Quintanilla 2005, p. 140) señala seis factores relevantes de la ciencia y la tecnología actuales para explicar el interés público, por lo que Quintanilla ha denominado la evaluación externa de tecnologías: 1) la rapidez del cambio científico-técnico, 2) la novedad de los problemas que el desarrollo científico-técnico plantea, 3) la complejidad e interdependencia de los proyectos tecnológicos, 4) la irreversibilidad de los efectos del desarrollo tecnológico en varios campos, 5) los problemas morales que suscitan las nuevas posibilidades tecnológicas, y, 6) la sensibilidad de la opinión pública ante los riesgos potenciales del desarrollo tecnológico.

${ }^{16}$ Fernando Broncano (ed.), op. cit., p. 15. 
Una comprensión clara de la incorporación de la tecnología en diferentes sectores de la vida social propiciará que las resistencias al cambio, al momento de incorporar este elemento, disminuyan, y si no hubiera tal resistencia el mismo conocimiento de ésta ayudará a su mejor aprovechamiento y, como consecuencia, el saldo será positivo para todos los actores involucrados en este proceso.

Una primera hipótesis es que hay resistencia por ignorancia, no por rechazo a la tecnología en sí. Es decir, desconocimiento del por qué y para qué de esa tecnología, y ante esta falta de conocimiento las personas se sienten amenazadas y desplazadas por la tecnología. No es objetivo de este apartado debatir las razones para tener esa percepción, sino la forma en la que se podría disminuir, en gran medida, esa resistencia. Si las personas comprenden las aplicaciones que la tecnología tiene en su ámbito personal y los beneficios que de ella se pueden generar habrá una aceptación inicial al uso de esta tecnología. ${ }^{17}$

Como expusimos antes, la democratización de la ciencia y la tecnología es un tema fundamental para atender nuestro problema. Para enfatizar esa importancia conviene distinguir entre los fines y los medios de la democratización. Este énfasis es especialmente importante si nos instalamos en la perspectiva de los países subdesarrollados. La siguiente es una revisión parcial de algunos autores que han escrito sobre la democratización de la ciencia, con el fin de tener un marco de referencia y apoyar los argumentos a favor de la gestión democrática de la tecnología.

Cabe la aclaración de que una de las principales tareas de las teorías contemporáneas sobre tecnología es establecer la relación entre ciencia y tecnología; sin embargo, la literatura al respecto es muy heterogénea y las contribuciones incluyen gran variedad de perspectivas. ${ }^{18}$ Una de las perspectivas que nos servirán para apoyar nuestras ideas es la de Feenberg quien asegura que no se ha podido llegar a un

${ }^{17}$ E. M. Rogers, Diffusion of Innovations, 1983, New York, The Free Press.

${ }^{18}$ Una revisión de dicha literatura nos llevó a identificar autores como Broncano (1995), Ordóñez (2003), Rubio (2003), Feemberg (1991), Olivé (2000). 
acuerdo en definir qué es ciencia y por lo tanto no se puede encontrar una distinción entre ciencia y tecnología. En un primer paso para lograr esta distinción Feenberg destaca tres aspectos de la ciencia:

1) La ciencia como una empresa social; 2) la ciencia conformada por leyes y teorías; y 3) la ciencia aplicada. De estas divisiones la segunda es central -leyes y teorías son el objeto de la ciencia como empresa social cuyo objetivo es proporcionar una representación de la realidad en exacta armonía con la naturaleza. Al hablar de ciencia aplicada se puede hacer referencia a la tecnología. ${ }^{19}$

Sin embargo, aclara este autor, pensar que la relación de la tecnología con la ciencia sólo se limita a entender tecnología como objeto - por sus aplicaciones- es dejar de lado ciertos factores importantes que tienen que ver con el contexto social en que se dan esas aplicaciones.

Por su parte, Olivé dice que la ciencia no se puede entender únicamente como un conjunto de teorías, ni la tecnología se entiende sólo como un conjunto de artefactos o de técnicas. Según esta concepción, la ciencia y la tecnología se entienden como constituidas por sistemas de acciones intencionales. ${ }^{20}$ Es decir como sistemas que incluyen a los agentes que deliberadamente buscan ciertos fines, en función de determinados intereses, para lo cual ponen en juego creencias, conocimientos, valores y normas.

De acuerdo con lo anterior, como la distinción entre ciencia y tecnología es casi imperceptible nosotros creemos que las preocupaciones que algunos autores han externado en relación a la necesidad de democratizar la ciencia se pueden adaptar sin mayor problema a la tecnología, ya que el principio de participación es equivalente en ambas actividades.

${ }^{19}$ A. Feenberg, Critical Theory of Technology, 1991, New York, Oxford University Press, p. 21.

${ }^{20}$ León Olivé, El bien, el mal y la razón. Facetas de la Ciencia y la Tecnología, 2000, Paidós, p. 86. 
En la Declaración de Santo Domingo ${ }^{21}$ "La ciencia para el siglo XXI: una nueva visión y un marco de acción" se plantean tres grandes metas para la democratización de la ciencia y la tecnología:

1. La ampliación del conjunto de seres humanos que se benefician directamente de los avances de la investigación científica y tecnológica, la cual debiera privilegiar los problemas de la población afectada por la pobreza;

2. La expansión del acceso a la ciencia, entendida como un componente central de la cultura;

3. El control social de la ciencia y la tecnología y su orientación a partir de opciones morales y políticas colectivas y explícitas.

Fuller ${ }^{22}$ propone pensar en la ciencia en el mismo sentido que nos referimos al concepto de democracia. Este autor dice que uno de los planteamientos que habría que hacer en relación a la ciencia es la forma de participación en la toma de decisiones que involucren varios intereses. Si este modelo (el de participación) se restringe a una élite, las divisiones internas de ésta proyectarían sus intereses particulares en la sociedad o bien, si este modelo puede ampliarse a la participación directa de todos, habría que imaginar el éxito o no del modelo en el mismo sentido que se plantea el éxito o no de una democracia. Si pensamos en sociedades pequeñas es un asunto menos complicado que cuando hablamos de sociedades más grandes y complejas.

Thus political theorists routinely query whether it makes sense to speak of 'democracy' once public participation is limited to the selection of representatives who, among themselves, legislate for the entire polity. ${ }^{23}$

${ }^{21}$ Reunión Regional de Consulta de América Latina y el Caribe de la Conferencia Mundial sobre la ciencia, auspiciada por la UNESCO, en Santo Domingo, República Dominicana, 10-12 de marzo de 1999. http://www.anuies.mx/principal/servicios/publicaciones/revsup/ res110/txt9_2.htm

${ }^{22}$ Steve Fuller, Science, 1997, Buckingham, Open University Press.

${ }^{23}$ Ibid., p. 3-4. 
Fuller propone la solución a este problema desde las ciencias sociales, con dos puntos de vista: la llamada Pequeña democracia y la Gran democracia. Mientras la principal ventaja de la Pequeña democracia es que sus integrantes comparten creencias y valores que los hace llegar a acuerdos consensuados, con los miembros de la Gran democracia, los intereses se contraponen sin pretender llegar al consenso.

Fuller explica que en los estudios contemporáneos sobre filosofía de la ciencia, la discusión sobre la pequeña y la gran democracia es similar al debate que se dio entre Thomas Khun y Karl Popper en 1965 en el cual el punto de la discusión era saber en dónde había más ciencia -en una sociedad cerrada o en una sociedad abierta. Con esta breve analogía, el autor inicia su tesis que considera a la ciencia ampliamente relacionada con la política y no únicamente como una parte constitutiva de ésta.

Siguiendo con este ejemplo, la pequeña democracia, que corresponde a la imagen kuhniana de la ciencia, ha estado históricamente alineada con el punto de vista de que una vez que los científicos acuerdan un paradigma que sirva como base para futuros cuestionamientos,

44 el mismo podría ser presentado a la sociedad en su conjunto como un asunto de expertos. De este modo, los científicos retienen su autoridad.

En contraste, la gran democracia relacionada con la imagen popperiana de la ciencia, defiende el punto de vista en el cual lo que los científicos toman como conocimiento es solamente un 'alto en el camino' hacia un cuestionamiento mayor y, por lo tanto, se podría esperar que los acuerdos realizados entre élites científicas puedan ser modificados, incluso cuestionados, hasta alcanzar las expectativas de comunidades específicas. Sin embargo, y esta es una parte medular en la tesis de Fuller, esta última posición no será posible si la contraparte de estas élites científicas no cuenta con el conocimiento necesario para debatir y argumentar sus posturas. 
Al parecer la discusión no termina; durante las pasadas dos décadas la sociología de la ciencia ${ }^{24}$ ha insistido en la necesidad de 'abrir los santuarios científicos', los laboratorios, e invitar a lectores inteligentes a juzgar lo que observan. Este discurso involucra temas sobre democracia y ciencia de forma simultánea. Los sociólogos de la ciencia se enfrentaron a un problema metodológico ya que ellos mismos pensaban en la ciencia como algo ‘ajeno' al resto de la sociedad; entonces era necesario cambiar esa actitud y empezar a 'incorporar la ciencia a la sociedad'. Fuller ilustra la propuesta de la sociología de la ciencia de la siguiente manera:

1. Si la gente acepta a la ciencia por lo que es más que por lo que no puede ser, ambas partes, científicos y sociedad, podrán tener una convivencia más sana. Una vez que se permita 'traspasar' las puertas de los laboratorios científicos podremos encontrar que el científico es alguien entrenado para hacer ciertas cosas útiles, en ciertos lugares que les permiten visualizar el mundo y realizar su trabajo de igual manera que cualquier otro profesionista.

2. La segunda perspectiva, más cercana a la posición marxista, se centra en la relación dialéctica entre lo que hacen los científicos y en lo que ellos (y otros) dicen que ellos hacen. Esta perspectiva empieza con el acuerdo con científicos en que el simple hecho de revelar el trabajo cotidiano del científico ayudaría más a despejar 'el misterio de su trabajo'.

A esta última perspectiva Fuller la denomina citizen-as-scientist por el papel que se le asigna a los grupos de 'no científicos' para autorizar el trabajo científico. A pesar de que esta perspectiva no es la más socorrida, es sin lugar a dudas, la más acorde con el ideal de 'transparencia democrática de la ciencia'

En lo que respecta a este ensayo, consideramos que los aspectos que destaca Fuller son igualmente válidos para los grupos de tecnólogos ya que si se conjuntaran los esfuerzos de éstos con los de la socie-

${ }^{24}$ Merton (1980), Fuller (1997), Brown, (2001), Feyerabend (1982), Mitcham (1989), Sonnert (2002). 
dad, sería crucial para equilibrar los intereses de ambos y lograr un desarrollo más eficiente de un grupo social determinado. Esto ayudaría a quitar esa imagen de que la tecnología -y quien la hace- es ajena a los intereses de la sociedad, cobijada siempre en 'la autoridad moral del conocimiento experto' ${ }^{25}$

Otra perspectiva es la de Andrew Ross, ${ }^{26}$ cuya propuesta para democratizar a la ciencia consiste en limitar 'el poder de los expertos' y dar mayor apertura a la 'gente común’ que tiene algún interés particular.

That may help to demystify, but it must be joined by insistence on methodological reform -to involve the local experience of users in the research process from the outset and to ensure that the process is shaped less by a manufacturer's interests than by the needs of communities affected by the product. Such methodological reform will lead from cultural relativism to social rationality. ${ }^{27}$

En el modelo de Ross se destaca la idea de compartir el poder entre el ciudadano común, que puede ser afectado por decisiones científicas, y el experto. Ross no aclara de qué manera se daría esta 'cooperación' para el mejor entendimiento de las necesidades de ambas partes y para ello Robert Brown propone dos formas de entenderlo. La primera forma de compartir el poder, sería que el 'no experto' informe al experto la manera en que ciertos productos o acciones pueden perjudicar su medio ambiente. La segunda aproximación para interpretar la propuesta de Ross, sería que ambas partes entraran en un proceso de negociación de lo que debe hacerse e intentar equilibrar intereses en juego, lo cual sería una forma de democratización de la ciencia bajo un modelo constructivista. Ninguna de las partes intenta llegar a la 'verdad absoluta' únicamente tratan de maximizar los beneficios.

${ }^{25}$ A. Pacy, The culture of Technology, 1984, Cambridge, MIT Press.

${ }^{26}$ Citado en Robert James Brown, Who Rules in Science, 2001, Cambridge, Harvard University Press.

${ }^{27}$ Ibidem, p. 171. 
Otra forma de concebir la democratización de la ciencia es la denominada Radical Science for the People Group,$^{28}$ que es un grupo de científicos y tecnólogos cuyo interés primordial son las relaciones de la ciencia con el orden social, con el firme propósito de poner a la ciencia al servicio de las clases oprimidas. El principal cuestionamiento a esta propuesta son los esquemas de financiamiento a proyectos, que de acuerdo con dicho grupo, generalmente están al servicio de la clase dominante. Brown ejemplifica esta problemática de la siguiente manera: se otorgan grandes sumas de dinero al estudio de problemas cardiacos o el cáncer, pero relativamente poco dinero a enfermedades asociadas con la desnutrición. No obstante y a pesar de los cuestionamientos, su estrategia define una serie de acciones positivas:

- Proveer asistencia técnica a las personas oprimidas. Incluye medicamentos, clínicas y asesoría de expertos en forma gratuita.

- Investigación de la actual estructura social.

- Desmitificación de la ciencia. Aquí la propuesta es proporcionar la información necesaria acerca de la ciencia y la tecnología para que la gente común pueda tener mayor control de su vida y pueda incidir en la toma de decisiones científicas cuando sus intereses se vean afectados por esas decisiones. ${ }^{29}$

En la propuesta se observan dos grandes ventajas: la primera es que este grupo (Radical Science for the People Group) no espera que se forme una sociedad democrática para lanzar sus iniciativas; proponen una ciencia más democrática que por medio de sus acciones ayude a conformar una sociedad más democrática. La segunda ventaja es la idea de que la ciencia ayuda; dentro de sus estrategias abordan asuntos que van desde la importancia de la salud física y mental, hasta el estudio de cómo funcionan ciertas estructuras sociales. Una vez que la comunidad maneja esta información pueden tener herramientas para mejorar sus condiciones de vida. Sin embargo, la misma comunidad

\footnotetext{
${ }^{28}$ Ibidem, p. 176.

${ }^{29}$ Ibidem.
} 
científica puede calificar de 'utópica' una propuesta de este tipo argumentando que a nadie le importa saber qué tanto una medicina o una técnica puede afectarles su vida cotidiana. La respuesta pragmática ('es utópico, por lo tanto hay que desecharlo') reduce la argumentación a cuestiones posibles pero cotidianas, que no generan la tensión necesaria para avanzar en la investigación.

Por su parte la preocupación de Harry Collins y Trevor Pinch está relacionada con la ciencia y la toma de decisiones tecnológicas en una sociedad democrática. Para estos autores es imprescindible que la sociedad sepa cómo funciona la ciencia como institución. La propuesta no es que el público conozca diversas teorías científicas, sino proporcionarle información de cómo se relaciona y trabaja la comunidad científica. En este sentido, la comunidad científica se tendría que abrir al gran público, incluso a la revisión de sus mecanismos y estructuras de financiamiento para las investigaciones.

Brown duda del interés de la gente común en comprender perfectamente cómo funcionan las instituciones científicas y propone como alternativa una democracia representativa en la cual los expertos actuarían en favor de los intereses de la comunidad. La propuesta es tener representantes cientificos por cada grupo relevante de la sociedad que tienen intereses en juego. La estrategia que propone Brown es tener un representante de cada grupo de la sociedad dentro de las comunidades científicas. Éstos serían los encargados de velar por la calidad de vida de su comunidad.

En la misma dirección que Brown, Feyerabend ${ }^{30}$ afirma que el juicio democrático rechaza 'la verdad' y la opinión de los expertos. Hay dos aspectos en esta pregunta. Uno se refiere a los derechos de los ciudadanos y de las tradiciones en una sociedad libre y el otro a las consecuencias de un ejercicio de estos derechos.

La tesis de Feyerabend es que en una democracia, un ciudadano tiene derecho a leer, escribir y hacer propaganda de cuanto despierte su fantasía. Si cae enfermo, tiene derecho a ser tratado de acuerdo a sus

${ }^{30}$ Paul Feyerabend, La Ciencia en una Sociedad Libre, México, Siglo XXI, 1982. 
deseos, bien por curanderos, bien por médicos. Y argumenta que sobre la base de este derecho, todo ciudadano tiene voz y voto acerca de la marcha de cualquier institución con la que coopere económicamente, bien sea con carácter particular o como contribuyente: colegios y universidades estatales y centros de investigación financiados por impuestos (como es el caso de la National Science Foundation o el CONACYT), deberían estar sujetos al criterio de los contribuyentes; lo mismo sucedería con todas las escuelas elementales de carácter local.

Al respecto, Feyerabend afirma que si los contribuyentes californianos quieren que en sus universidades estatales se enseñe vudú, medicina popular, astrología o ceremonias de la danza de la lluvia, esto sería lo que tengan que enseñar dichas universidades. La opinión de los expertos se tendrá en cuenta, pero la última palabra no la tendrán ellos. La última palabra provendrá de la decisión de comités democráticamente constituidos - como es la propuesta de Brown- en los cuales el hombre común tenga autoridad.

En este punto, Feyerabend lanza los mismos cuestionamientos que Brown: ¿posee el hombre de la calle los conocimientos precisos para tomar esta clase de decisiones? ¿No cometerá graves errores? ¿No resulta, por lo tanto, necesario dejar en manos de los expertos las decisiones más importantes? Y responde: en una democracia desde luego que no.

Una democracia es un colectivo de personas maduras y no un rebaño de ovejas guiado por una pequeña camarilla de sabelotodos. La madurez no se encuentra tirada por las calles sino que hay que aprenderla. No se aprende en las escuelas [...] sino por medio de una participación activa en las decisiones que se hayan de tomar. ${ }^{31}$

En este sentido, la participación del ciudadano común en las decisiones más importantes es necesaria aún cuando esto disminuya la cantidad de decisiones tomadas. Podemos concluir que la propuesta de todos los autores consiste en iniciar el proceso de cambio dentro de

${ }^{31}$ Ibid., p. 100. 
la ciencia y con ello ayudar a formar sociedades más democráticas en el futuro.

No se trata de tener 'eruditos' que hagan grupos cerrados sino que la sociedad cuente con la información necesaria para poder llegar a acuerdos y consensos con los expertos, en las áreas que se vayan a afectar por una decisión científica. A la luz de estos razonamientos valdría la pena retomar a Javier Ordoñez cuando se pregunta cómo caracterizar a la ciencia del siglo XXI pero desde nuestra época cultural:

Uno de los rasgos que ha caracterizado a la ciencia del siglo XX es la visibilidad. Este fenómeno no se da exclusivamente en la ciencia, pero en lo que a ella se refiere, muestra que está más presente que nunca en la sociedad, que actúa con voz propia y que las afirmaciones que contienen el adjetivo -científicas- tienen enorme importancia en todos los ámbitos de las relaciones sociales. [...] La ciencia se ha convertido en un valor social de gran importancia determinado por los contextos políticos y sociales. ${ }^{32}$

La argumentación anterior nos lleva a concluir que, bajo los conceptos modernos de desarrollo y el espacio de reflexión que se abre desde la filosofía de la tecnología, es imprescindible que los ciudadanos tengan la opción de participar en las decisiones tecnológicas que les atañen directamente y para ello es necesario que tengan conocimiento, no sólo de los aspectos tecnológicos básicos, sino de las consecuencias de la tecnología y de los mecanismos de participación. En este sentido, las decisiones tecnológicas no deben quedar sólo en manos de un grupo de expertos que ofrezcan soluciones 'mágicas' para los usuarios. También deberá haber expertos que faciliten la participación de las comunidades en la evaluación y toma de decisiones relacionadas con los problemas tecnológicos.

${ }^{32}$ Javier Ordóñez, Ciencia, Tecnología e Historia, 2003, México, FCE, p. 88. 